\title{
Clovis intentional bifacial overshot flaking: Two replica examples
}

\author{
Bruce Bradley
}

\section{Supplemental Information}

\section{Solutrean sample}

Well-documented large samples of Solutrean bifaces are generally not available for historical reasons. The sample used here was analyzed personally (with Dennis Stanford and Pegi Jodry). All pieces deemed complete enough to represent French Solutrean biface production technology were selected without regard to their physical traits. Pieces were not individually catalogued and did not have individual numbers, so are not listed as such in this table.

Table S-1. Solutrean* laurel leaf overshot flake scars

\begin{tabular}{llllll}
\hline Phase & 1 Face & 2 Faces & Present & Absent & Totals \\
\hline Early & $15(22.4 \%)$ & $8(40.0 \% \%)$ & $23(26.4 \%)$ & $16(15.2 \%)$ & 39 \\
Middle & $30(44.8 \%)$ & $6(30.0 \%)$ & $36(41.4 \%)$ & $30(28.6 \%)$ & 66 \\
Late & $13(19.4 \%)$ & $3(15.0 \%)$ & $16(18.4 \%)$ & $25(24.0 \%)$ & 41 \\
Finished** & $9(13.4 \%)$ & $3(15.0 \%)$ & $12(13.8 \%)$ & $34(32.4 \%)$ & 46 \\
\hline Totals & $\mathbf{6 7}$ & $\mathbf{2 0}$ & $\mathbf{8 7}$ & $\mathbf{1 0 5}$ & $\mathbf{1 9 2}$
\end{tabular}

* From the collections of Laugerie Haute West and Forneau du Diable, Musée National de Préhistoire, Les Eyzies-de-Tayac, France. Some pieces are fragmentary.

** Only includes those pieces considered not to have been reworked. 


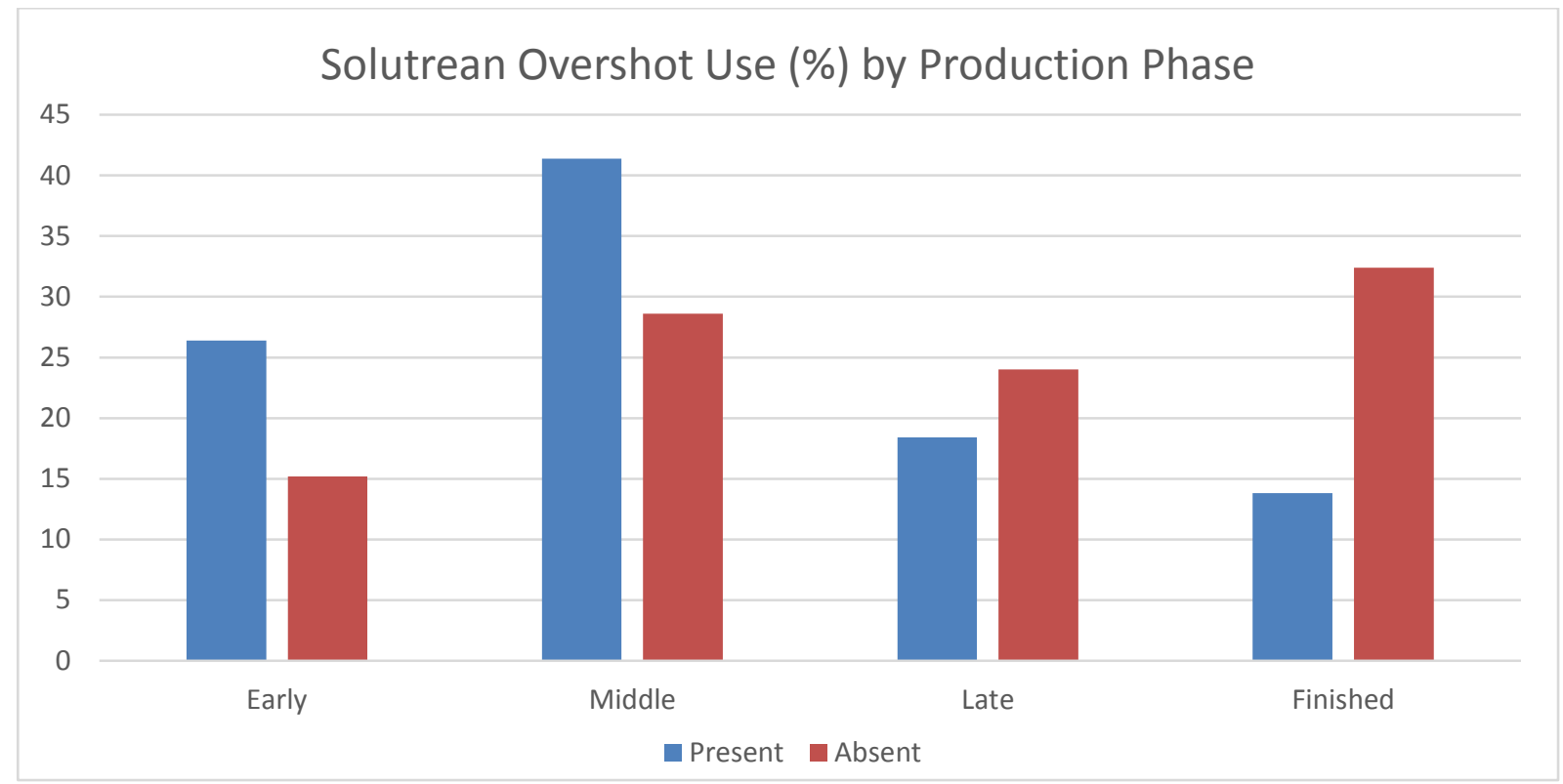

Figure S-1. Solutrean overshot use $(\%)$ by production phase.

Table S-2. Clovis* biface overshot flake scars.

\begin{tabular}{llllll}
\hline Phase & 1 Face & 2 Faces & Present & Absent & Totals \\
\hline Early & $12(11.3 \%)$ & $8(13.8 \%)$ & $20(12.2 \%)$ & $10(8.6 \%)$ & 30 \\
Middle & $45(42.5 \%)$ & $32(55.2 \%)$ & $77(47.0 \%)$ & $53(45.3 \%)$ & 130 \\
Late & $28(26.4 \%)$ & $12(20.7 \%)$ & $40(24.4 \%)$ & $35(29.9 \%)$ & 75 \\
Finished** & $21(19.8 \%)$ & $6(10.3 \%)$ & $27(16.5 \%)$ & $19(16.2 \%)$ & 46 \\
\hline Totals & $\mathbf{1 0 6}$ & $\mathbf{5 8}$ & $\mathbf{1 6 4}$ & $\mathbf{1 1 7}$ & $\mathbf{2 8 1}$ \\
\hline
\end{tabular}

* Counts derived from Bradley et al. (2010: table 3.1), Frison \& Bradley (1999), Waters \& Jennings (2015: table 23) and personal observation. All pieces were reclassified to conform with the reduction phases defined by Bradley et al. (2010:79- 106). See Bradley et al. (2010: table 3.3) for the relationships between this system and others.

** Only includes those pieces considered not to have been reworked.

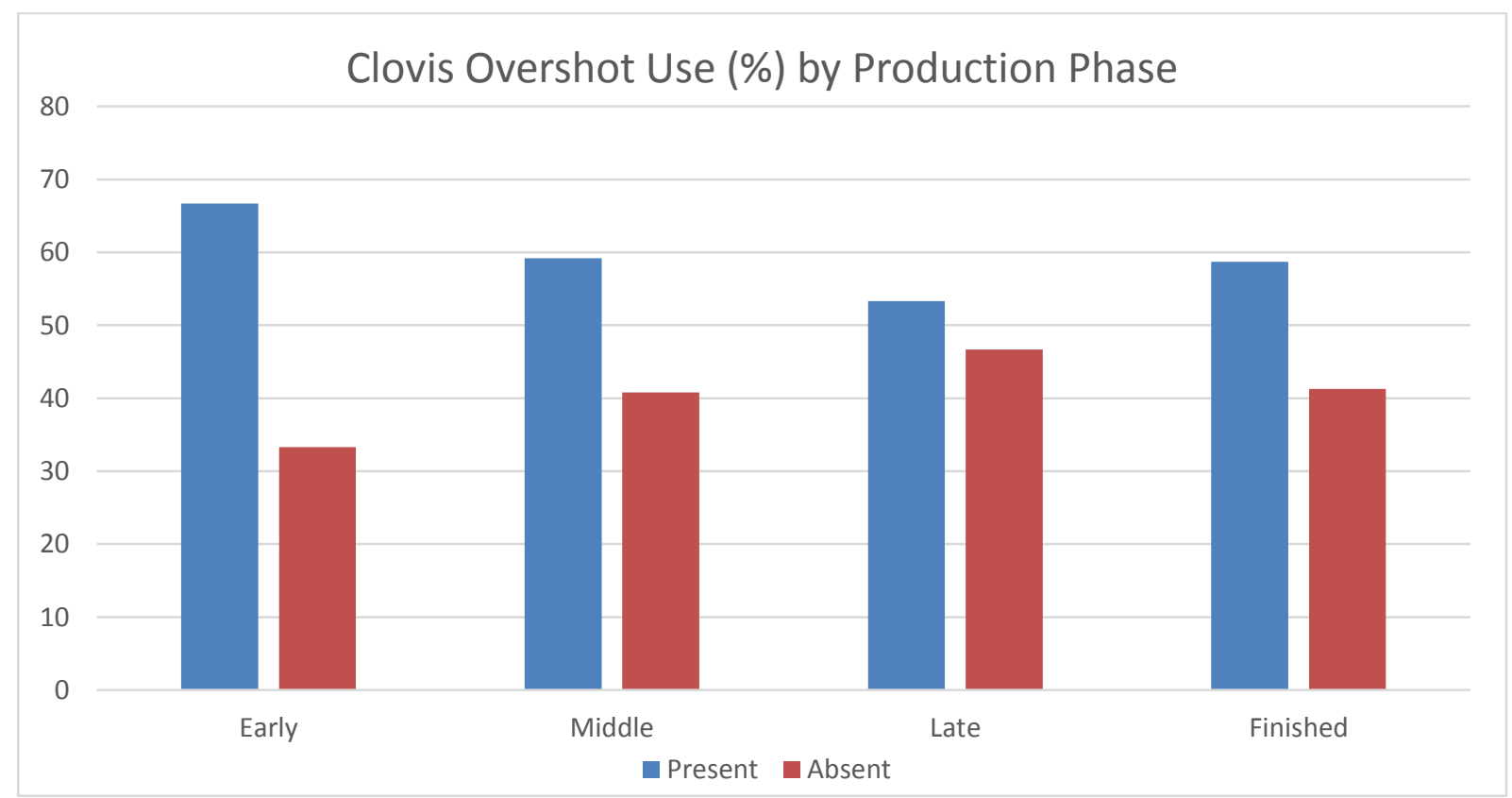

Figure S-2. Clover overshot use (\%) by production phase. 


\section{Clovis sample}

This sample was chosen to generally represent the range of Clovis technologies as seen at both a manufacturing site (Gault) and three widely spaced caches (Hogeye-Central Texas; Fenn-Northeast Utah and East Wenatchee - Central Washington). Caches were chosen because they have the greatest proportion of unfinished and unreworked examples. I recognize that they may also represent extraordinary pieces whose production technology may have been exceptional as well.

\begin{tabular}{|c|c|c|c|c|}
\hline \multirow[t]{2}{*}{ Assemblage } & \multicolumn{4}{|c|}{ Artifact numbers by production phase } \\
\hline & Early & Middle & Late & Finished \\
\hline $\begin{array}{l}\text { General Clovis, } \\
\text { (Bradley et al. } \\
\text { 2010:82-93) }\end{array}$ & \multicolumn{3}{|c|}{ See Tables $3.4,3.5$ and 3.6} & None \\
\hline $\begin{array}{l}\text { Fenn Cache, } \\
\text { (Frison and } \\
\text { Bradley 1999) }\end{array}$ & $\begin{array}{l}105,109 \\
110 \\
122,124\end{array}$ & $\begin{array}{l}101,102,103,104 \\
106,112,113,114 \\
115,116,117,118 \\
119,123,125,126 \\
127,128,129,130 \\
132,133,134,135\end{array}$ & $\begin{array}{l}106,131 \\
136,137\end{array}$ & $\begin{array}{l}107,120,121,138, \\
139,140,141,142, \\
143,144,145,146, \\
147,148,149,150, \\
153,154,155\end{array}$ \\
\hline $\begin{array}{l}\text { Hogeye Cache, } \\
\text { (Waters and } \\
\text { Jennings } 2015 \\
\text { Table 23) }\end{array}$ & None & $\begin{array}{l}1,5,10,11,15,25,26 \\
28.30,33,38,39,430 \\
41\end{array}$ & $\begin{array}{l}2,3,8,9, \\
12,14,16, \\
18,20,22, \\
23,24,29, \\
31,35,37, \\
42\end{array}$ & $\begin{array}{l}4,7,17,21,27,34 \\
43,44,45,46,47 \\
48,49,50,51,52, \\
53\end{array}$ \\
\hline $\begin{array}{l}\text { East Wenatchee } \\
\text { Cache (personal } \\
\text { analysis) }\end{array}$ & $\begin{array}{l}505,509 \\
514,515 \\
518,520\end{array}$ & $\begin{array}{l}321,510,511,512 \\
513,517\end{array}$ & 504,506 & $\begin{array}{l}325,326,327,328, \\
501,502,503,506, \\
507,508\end{array}$ \\
\hline
\end{tabular}

Table S-4. Fenn Cache overshot flake scars. Face 1 is on the left in the publication figures.

\begin{tabular}{lllll}
\hline & & \multicolumn{3}{l}{ Overshot Flake Scars } \\
\cline { 2 - 5 } Specimen\# & Phase & Face $\mathbf{1}$ & Face 2 & Total \\
\hline 105 & Early & 1 & 1 & 2 \\
109 & Early & 0 & 1 & 1 \\
110 & Early & 2 & 1 & 3 \\
122 & Early & 1 & 0 & 1 \\
124 & Early & 1 & 1 & 2 \\
\hline subtotal & & $\mathbf{5}$ & $\mathbf{4}$ & $\mathbf{9}$ \\
\hline 101 & Middle & 1 & 2 & 3 \\
102 & Middle & 1 & 2 & 3 \\
103 & Middle & 0 & 0 & 0 \\
104 & Middle & 3 & 1 & 4 \\
112 & Middle & 1 & 2 & 3 \\
113 & Middle & 0 & 0 & 0 \\
114 & Middle & 1 & 2 & 3 \\
\hline
\end{tabular}




\begin{tabular}{|c|c|c|c|c|}
\hline \multirow[b]{2}{*}{ Specimen\# } & \multirow[b]{2}{*}{ Phase } & \multicolumn{3}{|c|}{ Overshot Flake Scars } \\
\hline & & Face 1 & Face 2 & Total \\
\hline 115 & Middle & 1 & 0 & 1 \\
\hline 116 & Middle & 0 & 0 & 0 \\
\hline 117 & Middle & 0 & 0 & 0 \\
\hline 118 & Middle & 1 & 0 & 1 \\
\hline 119 & Middle & 0 & 0 & 0 \\
\hline 123 & Middle & 1 & 0 & 1 \\
\hline 125 & Middle & 0 & 0 & 0 \\
\hline 126 & Middle & 0 & 0 & 0 \\
\hline 127 & Middle & 0 & 1 & 1 \\
\hline 128 & Middle & 0 & 1 & 1 \\
\hline 129 & Middle & 0 & 0 & 0 \\
\hline 130 & Middle & 1 & 0 & 1 \\
\hline 132 & Middle & 0 & 0 & 0 \\
\hline 133 & Middle & 0 & 0 & 0 \\
\hline 134 & Middle & 0 & 0 & 0 \\
\hline 135 & Middle & 0 & 0 & 0 \\
\hline subtotal & & 11 & 11 & 22 \\
\hline 106 & Late & 0 & 0 & 0 \\
\hline 131 & Late & 0 & 1 & 1 \\
\hline 136 & Late & 1 & 0 & 1 \\
\hline 137 & Late & 0 & 1 & 1 \\
\hline subtotal & & 1 & 2 & 3 \\
\hline 107 & Finished & 0 & 1 & 1 \\
\hline 120 & Finished & 0 & 1 & 1 \\
\hline 121 & Finished & 0 & 0 & 0 \\
\hline 138 & Finished & 0 & 0 & 0 \\
\hline 139 & Finished & 1 & 1 & 2 \\
\hline 140 & Finished & 3 & 1 & 4 \\
\hline 141 & Finished & 0 & 0 & 0 \\
\hline 142 & Finished & 1 & 0 & 1 \\
\hline 143 & Finished & 1 & 1 & 2 \\
\hline 144 & Finished & 3 & 0 & 3 \\
\hline 145 & Finished & 3 & 2 & 5 \\
\hline 146 & Finished & 0 & 0 & 0 \\
\hline 147 & Finished & 1 & 0 & 1 \\
\hline 148 & Finished & 1 & 0 & 1 \\
\hline 149 & Finished & 1 & 1 & 2 \\
\hline 150 & Finished & 0 & 0 & 0 \\
\hline 153 & Finished & 1 & 1 & 2 \\
\hline 154 & Finished & 2 & 0 & 2 \\
\hline 155 & Finished & 0 & 0 & 0 \\
\hline subtotal & & 18 & 9 & 27 \\
\hline Totals & & 35 & 25 & 58 \\
\hline
\end{tabular}


Table S-5. East Wenatchee Cache overshot flake scars.

\begin{tabular}{|c|c|c|c|c|}
\hline \multirow[b]{2}{*}{ Specimen\# } & \multirow[b]{2}{*}{ Phase } & \multicolumn{3}{|c|}{ Overshot Flake Scars } \\
\hline & & Face 1 & Face 2 & Total \\
\hline 505 & Early & 0 & 2 & 2 \\
\hline 509 & Early & 1 & 0 & 1 \\
\hline 514 & Early & 0 & 0 & 0 \\
\hline 515 & Early & 0 & 0 & 0 \\
\hline 518 & Early & 1 & 0 & 1 \\
\hline 520 & Early & 0 & 0 & 0 \\
\hline subtotal & & 2 & 2 & 4 \\
\hline 321 & Middle & 0 & 2 & 2 \\
\hline 510 & Middle & 1 & 1 & 2 \\
\hline 511 & Middle & 1 & 0 & 1 \\
\hline 512 & Middle & 3 & 3 & 6 \\
\hline 513 & Middle & 0 & 0 & 0 \\
\hline 517 & Middle & 0 & 1 & 1 \\
\hline subtotal & & 5 & 7 & 12 \\
\hline 504 & Late & 0 & 3 & 3 \\
\hline 506 & Late & 2 & 0 & 2 \\
\hline subtotal & & 2 & 3 & 5 \\
\hline 4 & Finished & 0 & 3 & 3 \\
\hline 325 & Finished & 0 & 0 & 0 \\
\hline 326 & Finished & 0 & 4 & 4 \\
\hline 327 & Finished & 2 & 0 & 2 \\
\hline 328 & Finished & 1 & 0 & 1 \\
\hline 501 & Finished & 4 & 5 & 9 \\
\hline 502 & Finished & 1 & 0 & 1 \\
\hline 503 & Finished & 1 & 1 & 2 \\
\hline 506 & Finished & 2 & 1 & 3 \\
\hline 507 & Finished & 0 & 0 & 0 \\
\hline 508 & Finished & 0 & 0 & 0 \\
\hline subtotal & & 11 & 14 & 25 \\
\hline Totals & & 20 & 26 & 46 \\
\hline
\end{tabular}

